\title{
El enfoque sistémico en la evaluación de las unidades de información
}

\author{
Alvaro Quijano Solís \\ El Colegio de México, A.C.
}

\subsection{Resumen}

Dentro del marco conceptual del enfoque sistémico, se define y describe el proceso de evaluación de unidades de información como tarea que forma parte de la planificación general. Se conceptualizan los criterios o atributos que forman parte de este proceso, y finalmente, se concluye proponiendo un conjunto de requisitos previos y un enfoque integrador concretado en diferentes acciones que permiten llevar a cabo la evaluación de las unidades de información y de los productos generados por ellas.

Palabras clave: Planificación de unidades de información. Evaluación de unidades de información. Evaluación de productos de información. Teoría de sistemas.

\subsection{Abstract}

Inside the conceptual frame of the systemic approach, the process of evaluating information units is described as a task that is part of the general planning process. The criteria or attributes that affect this process are conceptualized. Finally, as a conclusion, a set of prerequisites and an integrated approach is proposed which, throughout different actions, allows the evaluation of the information units and their products.

Keywords: Information units planning. Information units evaluation. Information products evaluation. Systems theory. 


\section{Introducción}

Cada vez con mas frecuencia, se dedica un esfuerzo considerable a evaluar los resultados de unidades de información. Conforme nos aproximamos al establecimiento de prácticas profesionales más responsables y efectivas, los métodos para evaluarlas necesitan ser más formales, sistemáticos y, especialmente, generalizables para establecer criterios de clasificación en términos de medidas de efectividad y eficiencia.

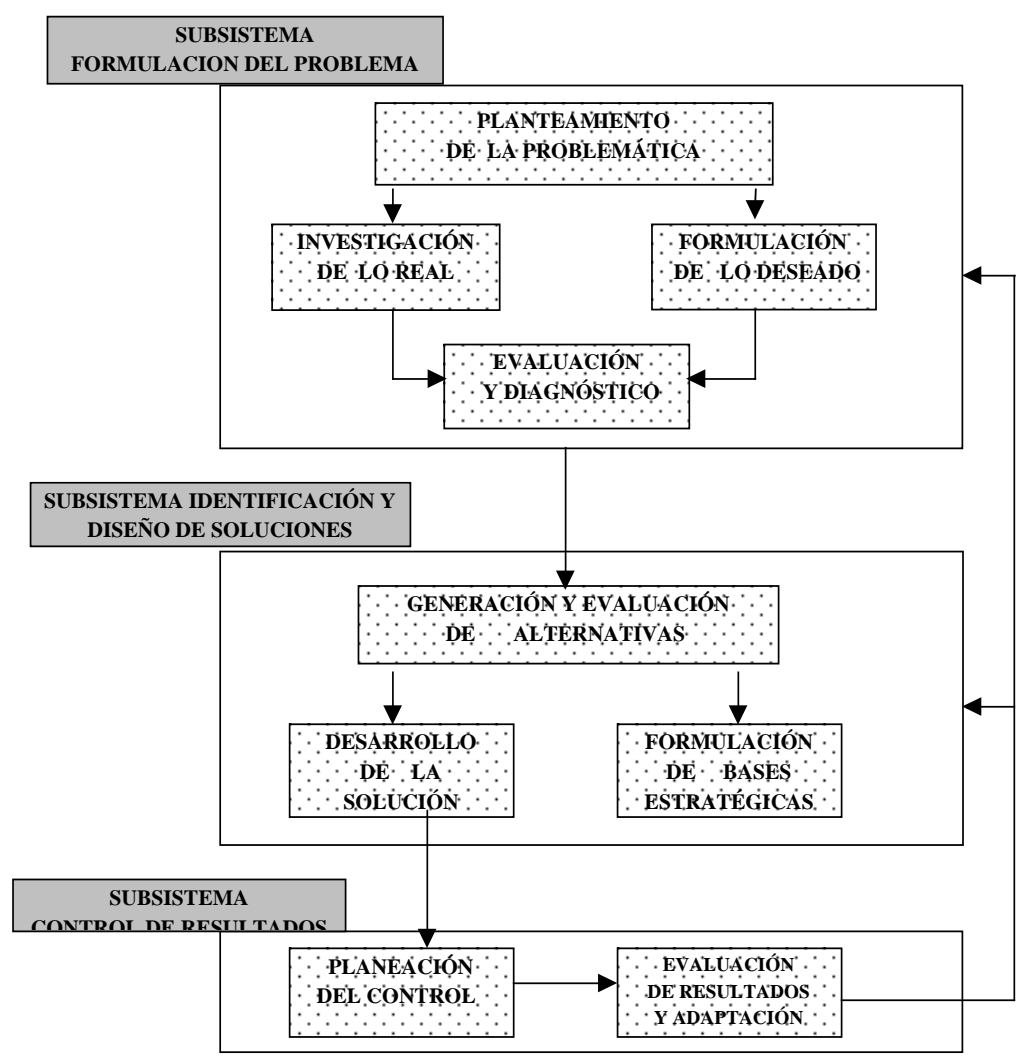

Fig. 1. Fuente: Fuentes Zenón, A. y G. Sánchez Guerrero. Metodología de la Planeación Normativa. Cuadernos de Planeación y Sistemas, 1. México: Universidad Nacional Autónoma de México, Facultad de Ingeniería, 1991. 
En contraste, los esquemas de atributos cuantitativos, como base de la evaluación, no resuelven los problemas planteados por el enfoque de una disciplina que está en una etapa descriptiva, mas que prescriptiva y que, por tanto, se encuentra en la búsqueda de un enfoque cualitativo, intrínsecamente relacionado con el concepto del "valor de la información" (Taylor, 1986 p.1-4).

El concepto mencionado se vuelve central en la tarea de evaluar unidades de información, toda vez que el enfoque de calidad, orientado al usuario, supone una valoración de costo-beneficio asociada al concepto de valor (Gerkes, 1998).

En resumen, el reconocimiento gradual de la importancia de los sistemas de información no ha ido acompañado de metodologías confiables para determinar su valor. En el mejor de los casos, se asume un grupo restringido de componentes del sistema sujeto a evaluación, mientras se ignoran otros. En este contexto, se hace necesario involucrar a todos los protagonistas que participan o son afectados por el proceso de transformar los insumos en productos en una unidad de información, en una negociación que construya alternativas consensuadas para resolver problemas planteados por la evaluación, con un enfoque holístico centrado en personas, mas que en tecnologías. Este último punto, que inspira el enfoque "socio-técnico" de Emery y Trist (Emery, Trist, 1960), es importante en la medida en que la velocidad de las habilidades humanas de aprendizaje es menor que la de surgimiento de nuevas tecnologías y, por tanto, se plantea un problema de asimilación tecnológica que incide directamente en las relaciones insumo/producto en las unidades de información (Quijano, Herrero, 1996) .

\section{Evaluación}

En este trabajo se describen algunas ideas del enfoque sistémico aplicables al proceso de evaluación de unidades de información, se retoman criterios de calidad para evaluar a éstas y se explica el marco conceptual del proceso evaluativo, desde la perspectiva de los sistemas suaves.

En una primera aproximación, podría definirse la evaluación de una unidad de información como la aplicación sistemática de los procedimientos de investigación a la valoración del concepto, diseño, implantación y utilidad de una unidad de información. Se trata, pues, de establecer juicios de valor sobre el proceso que va desde la definición hasta la implantación de una unidad de información. Esta definición, inspirada en la de Rossi y Freeman (1989), considera la evaluación en tres momentos: la evaluación diagnóstica, antes de la planeación de una solución; la evaluación ex-ante, que se efectúa antes de implantarla y la ex-post, que se realiza después de obtener resultados. La distinción es pertinente, toda vez que, en el uso común del término evaluación, generalmente solo nos referimos a

Scire. $5: 1$ (en.-jun. 1999) 27-39. 
la evaluación ex-post, ya que es la que arroja elementos objetivos en la forma de resultados.

Si se acepta la definición propuesta como primera aproximación, se sigue que la evaluación es parte de la planeación y, por tanto, constituye en sí misma un sistema compuesto por la evaluación diagnóstica, la ex-ante y la ex-post.

La evaluación es, entonces, un proceso continuo, a lo largo del propio proceso de planeación, toda vez que el objeto a evaluarse, las unidades de información, responden a un entorno dinámico y los productos de ellas son altamente sensibles a la valoración del usuario y sus insumos lo son también, al provenir de ambientes informativos en cambio permanente. De esta manera, la toma de decisiones que se apoya en la evaluación, igual que la solución de problemas en esta área, debe ser un proceso continuo que, al estar sujeto a múltiples influencias de los flujos sociales de la información, debe incorporar en la evaluación a los múltiples componentes de la unidad de información con un enfoque integrador y, por tanto, sistémico (1).

Las reflexiones anteriores nos llevan a pensar que no sería factible, en la mayor parte de los casos, esperar que una sola persona pudiese afrontar la tarea de evaluar una unidad de información y determinar, para ello, el valor de esta unidad.

Avgerou sugiere que la evaluación hecha por "expertos" contradice el reconocimiento prevaleciente de que el proceso de desarrollo de los sistemas de información involucra comportamientos políticos y, por otro lado, ignora el hecho de que los sistemas de información participan, cada vez más, como actores de los procesos estratégicos de toma de decisiones (Avgerou, 1995). Estas dos dimensiones se aúnan a la ya tradicional del proceso de evaluación como estimación de costo-beneficio y, en conjunto, constituyen el sistema de enfoques en el que se inscribe el proceso de evaluación de unidades de información: dimensión económica, dimensión política y dimensión estratégica.

A manera de resumen de este apartado, en la figura 1 se presenta una conceptualización sistémica del proceso de planeación y en donde se pueden apreciar las intervenciones subsecuentes del subsistema de evaluación, a lo largo del proceso de planeación normativa.

\section{Conceptualización de atributos}

En la mayor parte de la literatura sobre evaluación de unidades de información, puede distinguirse una tendencia a considerar que los atributos o criterios que deben emplearse en los procesos de evaluación pueden agruparse en cinco conceptos principales: 
1. Cobertura. Se refiere a la proporción que alcanza el servicio respecto a la población atendida.

2. Efectividad. Es un concepto multidimensional que se refiere al grado en que se satisfacen objetivos y metas de la unidad de información.

3. Eficiencia. Se refiere al costo de satisfacer las metas y objetivos, en función de los recursos.

4. Costo en relación a la efectividad o a los beneficios. Se refiere a los estudios para contrastar el impacto de un servicio, programa o actividad, con los costos.

5. Calidad. Basado en el supuesto de que pueden alinearse las filosofías, actitudes y conductas de los componentes de la unidad de información para buscar la mayor satisfacción de los usuarios (Hernon, Altman, 1996, p.14).

Tradicionalmente, las bibliotecas y otras unidades de información se han centrado en los primeros tres conceptos. Ahí se incluyen los conteos de las actividades y procesos asociados con la organización de la información, así como los de las transacciones realizadas, la disponibilidad de materiales, etc.

El tratamiento de estos conceptos en la literatura ha sido extenso y no los estudiaremos en este trabajo por el hecho de que corresponden a una parte de la evaluación que se ajusta, casi siempre, a una necesidad administrativa de legitimar la actividad de la unidad de información mostrando resultados que permitan justificar su existencia ante las instancias que proporcionan los recursos. De esta manera, muchos de estos factores se han incluido en normas nacionales e internacionales y, en algunos casos, constituyen un elemento de clasificación presupuestal.

Nos concentraremos, pues, en los dos conceptos asociados con costo y calidad por ser los mas relacionados con los procesos de evaluación en sus tres dimensiones: económica, política y estratégica. Además, es en ellos en donde los procesos de intervención deben hacerse en un marco sistémico y, particularmente, participativo entre los diferentes actores de una unidad de información. Obviamente, no debemos olvidar que estos dos factores deben considerarse en conjunto con los mas cuantitativos, si se pretende que la evaluación sea holística y que se acerque mas al comportamiento real de la unidad a evaluarse.

\section{Valoración de atributos}

Como Taylor afirma, el valor de la información no puede ser medido en términos precisos antes de que sea usada, contextualizada (Taylor, 1986, p. 203). Así, mientras mejor entendamos el ambiente en el que se usa la información, 
estaremos mas capacitados para estimar su valor y mejorar a la unidad de información. Dicho de otra manera, la evaluación supone un proceso de negociación entre el sistema de información y el entorno, incluyendo a sus usuarios.

De hecho, en su modelo de valor agregado Taylor propone que un sistema de información debe ser visto como un conjunto de prácticas sociales formales que contiene tres elementos:

- un sistema formal de procesos que agregan valor a datos.

- uno o más conjuntos de usuarios que establecen sus criterios para juzgar la utilidad de la información.

- un espacio de negociación entre sistema y usuarios, en donde el sistema intenta ayudar a los usuarios en sus procesos de toma de decisiones (Taylor, 1986, 202).

En general, puede asegurarse que el proceso de toma de decisiones (académicas, económicas, estratégicas, etc.) de los usuarios, atiende a una racionalización de esfuerzos (dinero, tiempo, recursos, etc.) para decidir la información o producto de información que más se acerca a sus propios objetivos (de estudio, de investigación, de negocios, etc.) Sin embargo, la racionalización que el usuario hace no resulta tan sofisticada y, en general, obedece a un sentido común basado en la experiencia (Loasby, 1976).

Taylor propuso seis categorías para agrupar los criterios que, en su opinión, el usuario utiliza (consciente o inconscientemente) para escoger la información de entre una variedad de opciones:

1. Facilidad de uso (o de acceso, por ejemplo)

2. Reducción de ruido (control de vocabulario, por ejemplo)

3. Calidad (exactitud, actualización, confiabilidad, validez, etc.)

4. Adaptabilidad al problema (cercanía, flexibilidad, simplicidad, etc.)

5. Ahorro de tiempo (velocidad de respuesta, por ejemplo)

6. Ahorro de costos (tiempos de conexión, formatos, etc.) (Taylor, 1986, p. 50)

Otros autores han abundado sobre criterios de evaluación en sistemas de información y la literatura es profusa en enfoques teóricos, mas que pragmáticos, aunque parece haber un consenso en usar la evaluación del servicio como piedra angular de la calidad y de los costos. Así, Hernon y Altman han desarrollado un instrumento de encuesta para establecer un marco a la calidad del servicio en unidades de información.

El esquema que proponen puede resumirse de la siguiente manera:

I. Contenido informativo

Scire. $5: 1$ (en.-jun. 1999) 27-39. 


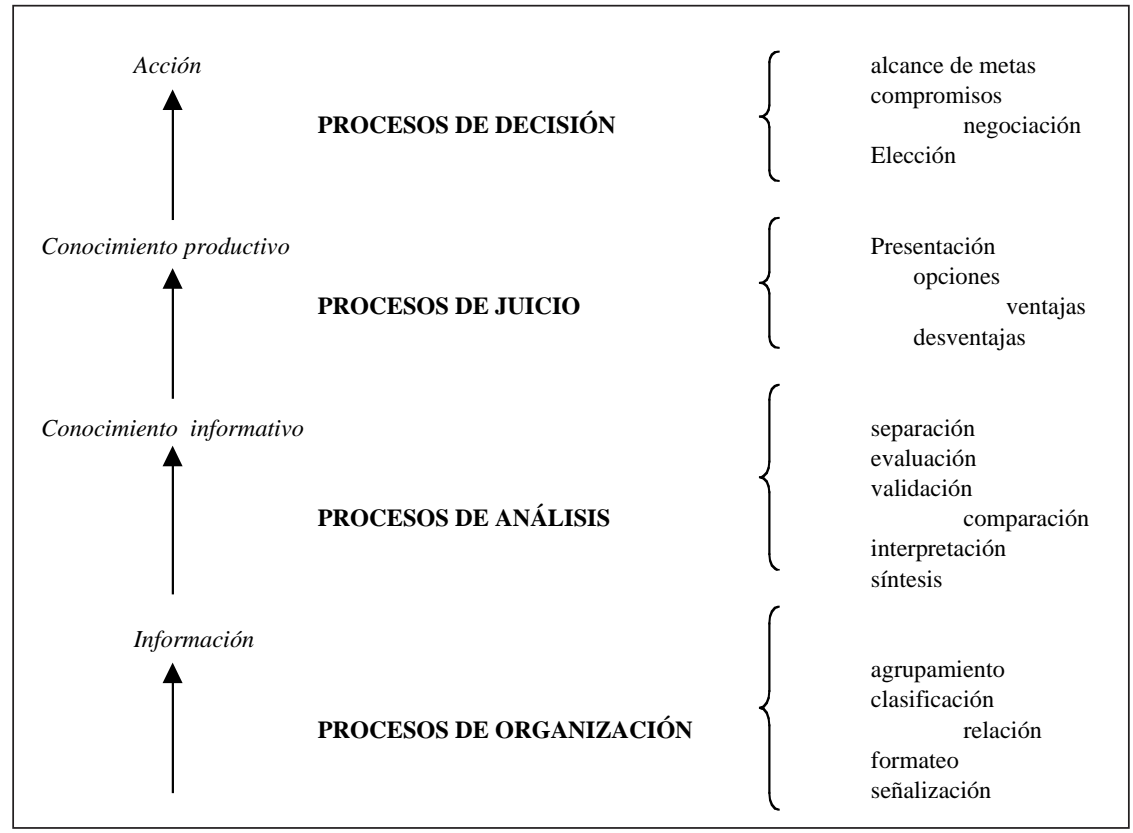

Fig. 2. Criterios según Taylor

1.1 Precisión en la respuesta

1.2 Exactitud en las fuentes

1.3 Actualización

1.4 Comprensión

\subsection{Formatos}

1.6 Estética de la presentación

1.7 Pertinencia de la información

II. Organización, ambiente y entrega de la información

2.1 Accesibilidad

2.2 Capacidad de respuesta a quejas

2.3 Condiciones físicas del material (para su disponibilidad)

2.4 Ambiente físico

2.5 Costos

2.6 Reputación de servicio

Scire. $5: 1$ (en.-jun. 1999) 27-39. 
III. Personal de servicio

3.1 Capacidad de educación de usuarios

3.2 Precisión de respuestas

3.3 Capacidad de comunicación

3.4 Actitud de servicio

3.5 Conocimientos

3.6 Velocidad de respuesta

3.7 Disponibilidad en tiempo

IV. Personal técnico y de sistemas

4.1 Habilidad para comunicarse con otros miembros del personal

4.2 Precisión de respuestas

4.3 Anticipación a las necesidades del usuario

4.4 Conocimiento de normas técnicas

4.5 Orientación al servicio

4.6 Velocidad de respuesta

4.7 Conocimiento tecnológico (Hernon, Altman, 1996, p. 66-67)

La selección de criterios debe ser una tarea que, al tiempo que se inserta en la cadena concepto-categoría-variable-criterio-indicador-norma, supone un alto grado de abstracción sistémica para proponer algo mas integrado que un conjunto no relacionado de indicadores puntuales. Se trata de un proceso que supone un marco teórico y que desemboca en indicadores pertinentes para la evaluación de unidades de información.

El proceso de establecer criterios supone una visión del sistema a evaluar, de su medio ambiente o suprasistema, y de las relaciones funcionales y estructurales entre ambos. Se trata, pues, de reconocer la ecología social y organizacional de la unidad de información: cómo es afectada por su contexto y en qué lo afecta. Por ello, la evaluación de una unidad de información debe darse en el marco de una visión compartida entre los diferentes actores comprometidos.

Los dos esquemas presentados derivan de dos puntos de vista complementarios. El enfoque de Taylor propone conceptos emanados de su análisis intrínseco del valor de la información y de su percepción del punto de vista del usuario, tal como se esquematiza en la figura 2 (en la página anterior). Es importante resaltar que, en este esquema, los datos se convierten en información cuando se establecen relaciones entre ellos. (Taylor, 1986, p. 6) 
Por su parte, el esquema de Hernon y Altman borda alrededor del concepto de calidad total y está validado por un consenso entre bibliotecarios de cinco bibliotecas académicas norteamericanas.

Los resultados de ambos enfoques permiten construir un grupo común de variables involucradas en el proceso de evaluación de unidades de información, propuesta que no se presenta en este trabajo por cuanto no es parte de sus objetivos (Quijano, Vega, 1998).

\section{Un enfoque unificador}

Existe una disyuntiva aparente entre los criterios de calidad y cantidad, así como entre la evaluación objetiva y la subjetiva. Se pueden, también, detectar dificultades provenientes de los objetivos intangibles que juegan un papel político determinante en la creación y operación de unidades de información y cuya evaluación difícilmente puede cuantificarse. Finalmente, se sabe que los efectos esperados de las tecnologías de información (mayor productividad, ahorros sustanciales, etc.) dependen en mucho de características sociotécnicas, asociadas con la aceptación tecnológica y con la estructura de la calidad de vida en el trabajo, características que, a su vez, están sujetas a cambio permanente durante la vida del sistema (Avgerou, 1995, p.430).

A pesar del énfasis reciente en el aprendizaje de la organización y su relación con la información y su correspondiente "aprender a aprender" en las organizaciones educativas, estos factores están ausentes de los esquemas evaluativos, que favorecen enfoque cuantitativos y cualitativos, al margen de los impactos sociales y organizacionales.

Un enfoque de sistemas suaves que ha sido desarrollado para el área de sistemas de información con el objetivo de lograr un mejor entendimiento de los requisitos que debe tener un sistema de información, a partir de un proceso permanente de negociación y construcción de soluciones, se resume en el libro de Checkland: Information, systems and information systems (Checkland, Holwell, 1998).

El enfoque de sistemas suaves de Checkland favorece la visión del valor de los sistemas de información desde la perspectiva de los involucrados (stakeholders) y, de esta manera, los costos, beneficios y otros aspectos, toman su lugar en el proceso de evaluación como soportes de la negociación entre los involucrados.

Guba y Lincoln (1989) presentan la argumentación ontológica y epistemológica de este método de evaluación, a partir de la evaluación educativa, para justificar el cambio de los métodos científicos de búsqueda de verdades objetivas, hacia un método de búsqueda y de entendimiento basado en la exploración de las

Scire. $5: 1$ (en.-jun. 1999) 27-39. 
"construcciones" del pensamiento de los involucrados en un proceso de evaluación.

El método se basa en el supuesto de que hay posibilidad de consenso, basado en el entendimiento de los valores de cada participante y que se pueden establecer construcciones conjuntas, a través de un proceso dialéctico, buscando resolver los conflictos a partir de los cambios propuestos al sistema de información.

Así, para Checkland, las tareas del analista deben ser:

1. Entender las diferentes visiones (construcciones) y construir modelos alternativos de la situación que las incorporen.

2. Usar esos modelos para diagnosticar la situación.

3. Facilitar el consenso entre los involucrados sobre un conjunto de acciones que establezca los requerimientos del cambio (2).

Como un pequeño ejemplo, en el caso de nuestra biblioteca, desde 1996 se inició un proceso de cambio en las actividades del personal, mas como una reacción al impacto de las innovaciones tecnológicas que como un proceso planeado (Quijano, Vega, 1998). El resultado ha sido el malestar organizacional que se produce por la multitud de tareas a desarrollar, que contrastan con un deseo exacerbado de no abandonar ninguna, lo que provoca una sobresaturación del personal, y la sensación de frustración.

A fines de 1998, se inició un proceso de evaluación de la situación descrita, para implementar posibles cambios. Se levantaron las opiniones de los 15 profesionales que no tienen cargo administrativo. Las encuestadoras prepararon un resumen incluyendo todas las opiniones sin ponderarlas y, sobre este, otros dos profesionales, que no participaron en la encuesta, prepararon un primer documento de cambios propuestos, sobre el que el director deberá preparar el documento sobre el cual iniciar un proceso de negociación con todos los involucrados.

Paralelamente, dos encuestados en la primera ronda se convierten en encuestadores para recabar, a su vez, las opiniones de los profesionales que tienen cargo administrativo, incluyendo al director.

Se espera que, de este proceso, surja el plan de trabajo para el próximo lustro, que modifique sustancialmente la forma en la que se viene trabajando. El enfoque propuesto determina el contenido de la evaluación (criterios) en función de su contexto (qué interesa a los participantes), y permitirá establecer el contexto global de la organización a partir de consensuar valores. El principal beneficio de este proceso es el involucramiento de los participantes en un intercambio informado de preocupaciones, quejas y visiones. 
Los procesos participativos buscan, en primera instancia, la legitimación del proceso de cambio, y al involucrar a los participantes, se convierte en un proceso educativo que precisa la validez de las quejas en un ambiente dialéctico de negociación.

Como Avgerou sugiere, el principal problema de este enfoque proviene del posible choque entre los estilos de administración y la forma de percibir el liderazgo (Avgerou, 195, p.436). En nuestra experiencia, este problema se manifiesta mas en un entorno de discusión colectiva, y es por esta razón que se utilizó, en la primera etapa, una técnica de entrevista, para evitar el choque frontal. De paso, se permitió la expresión de aquellos participantes que, usualmente, no expresan su opinión en público.

\section{A modo de conclusión}

El enfoque propuesto en la literatura revisada puede centrarse en los siguientes puntos:

1. Identificación de todos los involucrados

2. Detección, en cada uno, sus construcciones, preocupaciones y quejas.

3. Proponer decisiones que puedan ser comprehensivas y consensuales.

4. Probarlas y aumentarlas con nueva información.

5. Preparar la agenda de negociación.

6. Proveer la información que apoya la agenda.

7. Negociar y reiniciar el ciclo si persisten conflictos.

Guba y Lincoln (1989) establecen cuatro condiciones para este proceso:

1. Compromiso de los involucrados en alcanzar consenso.

2. Capacidad para negociar.

3. Deseo de compartir el poder.

4. Deseo de reconsiderar las posiciones personales y de comprometerse con las acciones consensuadas.

Los atributos propuestos en la primera parte de este documento, constituyen una plataforma inicial para negociar los criterios de evaluación, de una manera similar a la que, en nuestra experiencia, hemos realizado para consensuar la evaluación de nuestra biblioteca. Por supuesto, en la evaluación de las unidades de información y de sus productos, debe involucrarse a otros dos sectores que tienen la capacidad de ser "propietarios" del sistema: los usuarios y los directivos de la organización a la que, por lo general, pertenece la unidad de información. 
Si bien se trata de un proceso más amplio de negociación, se cree que las líneas mas generales son las mismas que las descritas anteriormente.

\section{Notas}

(1) Cfr. Sánchez Guerrero, G. (1994) En esta obra, se propone la siguiente definición: La evaluación es un proceso de indagación mediante el cual se comparan los aspectos relevantes del sistema contra los fines y un patrón establecido, obteniéndose una valoración, cuantitativa y/o cualitativa, mediante una ordenación preferencial y produciendo recomendaciones para que el sistema se acerque a los objetivos previstos (p. 76). La adaptación es mía y la definición sugerida asume que la evaluación es en sí misma un proceso de transformación de un insumo formado por el reconocimiento de los aspectos relevantes del sistema, en un producto que es la base de la toma. El proceso de indagación debe ser, según el autor, holístico, continuo, participativo, plural, preventivo y contingente.

(2) Como se indicó anteriormente, al resultado de esta síntesis entre ambos enfoques está mas orientada a aspectos cualitativos (aunque algunos derivan en cuantitativos). Cualquier esquema de este tipo estaría incompleto si no considerara variables cuantitativas como número de transacciones por usuario, porcentaje de ocupación de espacio, etc. Para estos efectos existen fuentes como la American Library Association (ALA), la Association for College and Research Libraries (ACRL) y la International Federation of Library Associations and Institutions (IFLA), que han desarrollado normas cuantitativas. Sin embargo, como ya se ha apuntado, la tendencia se mueve hacia normas de aseguramiento de calidad, de las que las ISO-9000 son un ejemplo

\section{Bibliografía}

Avgerou, C. (1995). Evaluating information systems by consultation and negotiation. // International Journal of Information Management, 15 : 6 (1995) 427

Checkland, P.; Holwell,S. (1998). Information, systems and information systems. London : Wiley, 1998.

Emery, F.E.; Trist E.L. (1960). Socio-Technical systems. // C.W. Churchman ; M. Verhulst (eds) Management science, models and techniques. Vol. 2, Pergamon, 1960.

Gerkes (1998). Application of systems engineering in quality assurance. // ASLIB Proceedings. $50: 7$ (1998) 141

Guba, E. ; Lincoln, Y.(1989) Fourth generation evaluation. Los Angeles : Sage, 1989.

Hernon, P. ; E. Altman (1996). Service quality in academic libraries. New Jersey : Ablex, 1996 , p.19

Loasby, B.J.(1976). Choice, complexity and ignorance: an inquiry into economic theory and the practice of decision making. New York : Cambridge, 1976.

Quijano A. ; V. Herrero. (1996) Informatización y perfil bibliotecario : el caso de la Biblioteca de El Colegio de México. // Conferencia internacional de Bibliotecas de Educación Superior. Santiago de Chile : Colegio de Bibliotecarios de Chile, 1996. 
Quijano, A. ; Vega, G. (1998).Valor agregado y desarrollo de habilidades informativas: un enfoque sistémico. // Referencias. 3 : 2 (Agosto, 1998) 44-50

Rossi, P. ; Freeman H.(1989). Evaluation : a systematic approach. Beverly Hills : Sage, 4 ed., c1989.

Sánchez Guerrero, G.(1994). Evaluación de programas sociales : enfoque a programas académicos universitarios. México : Universidad Nacional Autónoma de México, Facultad de Ingeniería, 1994

Taylor, R.S. (1986). Value-added processes in information systems. New Jersey : Ablex, c1986. p. 1-4. 